\title{
A WEIGHTED MIN-NORM METHOD WITH A RECURSIVE IMPLEMENTATION
}

\author{
M. D. Ortigueira and M.A. Lagunas
}

TSC, ETSIT, Universidad Politecnica de Cataluña

Apdo 30002, 08080 Barcelona, Spain

\section{ABSTRACT:}

Min-norm method is related to MUSIC. This relation suggests us a weighted version of min-norm method obtained from the EV method of Johnson and DeGraaf. This new method is obtained by weighting the eigenvectors with the inverse of the eigenvalues. Some experimental results will illustrate the comparative behaviour of the algorithm. Both methods were implemented with a new recursive algorithm. This algorithm performs the spectral decomposition of the correlation matrix and is based on the properties of the elemental matrices $\mathbf{v} \cdot \mathbf{v}^{\mathrm{H}}$ called dyads. The problem is reduced to an eigendecomposition of the sum of a diagonal matrix with a dyad, which is easily obtained. This computation can be executed in a high degree of parallelism.

\section{1 - INTRODUCTION}

Min-norm method [3] is a well known high resolution method. In this paper we relate it with MUSIC [10]. This relation suggests us a weighted version of min-norm method obtained from the EV method of Johnson and DeGraaf [2]. This new method is presented in sec. 2 and it is essentially the min-norm method but with the eigenvectors weighted by the inverse of the eigenvalues. Preliminary results show that this method has a slightly better performance than the usual. In particular the zeros corresponding to existing sources are nearer the unit circle. Some experimental results will illustrate the comparative behaviour of the algorithms.

Both methods were implemented with a new recursive algorithm we propose also. This algorithm is presented in $\sec 3$ and it performs the spectral decomposition of the correlation matrix we assume to be recursively generated

* On leave from INESC and Instituto Superior Técnico, Lisbon, Portugal.

This work was supported in part by National Research Plan of Spain (PRONTIC-89) according to:

$$
\mathbf{R}_{\mathrm{N}}=\alpha \cdot \mathbf{R}_{\mathrm{N} \cdot 1}+(1-\alpha) \cdot \mathbf{v} \cdot \mathrm{v}^{\mathrm{H}}
$$

There are some solutions to the problem. The most important are described in [9] and [11]. We are going to propose an algorithm wich is equivalent to that described in [11] but with a very different formalism [ $5,6,7]$.

The algorithm is based on the properties of the elemental matrices $v . v^{H}$ called dyads [8]. To solve the posed problem we reduce it to an easier one by expressing the dyad in terms of the previous eigenvectors. The new problem becomes simply the spectral decomposition of the sum of a diagonal matrix with a dyad. The eigenvectors of this new matrix are easily computed with only 1 real and 1 complex operation by component and the eigenvalues are the zeros of a rational function whose partial fraction decomposition is known. These zeros are located inside disjoint intervals which allows us to obtain good starting values to the iterative Newton-like algorithm we use. This computation can be executed in a parallel way. The results of this algorithm are similar to those obtained with conventional algorithms. On the other hand we did not observe any numerical degradation with the increase in the number of dyads.

\section{2.- A WEIGHTED MIN-NORM METHOD}

The MUSIC bearing estimation method proposed by Schmidt [10] is based on the fact that the signal spatial covariance matrix $R_{X}$ has rank equal to the number of sources $(\mathrm{N})$. Thus, the spatial covariance matrix of the observed data $R_{x}$ under white noise assumption is given by

$$
\mathbf{R}_{\mathrm{x}}=\mathbf{R}_{\mathrm{s}}+\sigma_{\mathrm{n}}^{2} \mathbf{I}
$$

where $I$ is the identity matrix and $\sigma_{n}^{2}$ is the white noise variance. Letting $v_{n}=\left[v^{N+1}, \ldots, v^{L}\right]$ be the matrix span of the noise subspace and a the steering vector :

$$
a=\left[1, e^{j \omega}, e^{2 j \omega}, \ldots, e^{j L \omega}\right]^{\top}
$$


MUSIC method identifies the bearing estimates as the location of the peaks in the spectral estimate :

$$
P(\omega)=\left[a^{H} V_{n} v_{n}^{H} a\right]^{-1}
$$

The MIN-NORM method [3] uses a vector $d^{1}$ spaning the whole noise subspace of $\mathbf{R}_{\mathbf{x}}$. Its first element is constrained to be unity and its Euclidean length is required to be minimum. The bearing estimates are obtained from the zeros of

$$
Q(z)=\sum_{1}^{L} d_{i}^{1} \cdot z^{i-1}
$$

with $\mathbf{d}^{1}$ given by

$$
d^{1}=\left[\begin{array}{ll}
\ldots 1 & \ldots \\
v_{n}^{1} u / u^{H} &
\end{array}\right]
$$

where $v_{n}^{1}$ is obtained from $v_{n}$ by deleting the first line $u^{H}$.

The normalization of the first component to unity is not required; we can fix its value as being equal to the square norm of $u$. Then the Min-Norm polynomial coeficients may be given by:

$$
d^{1}=v_{n} \cdot u^{1}
$$

where $u^{1}$ is the transpose conjugate of the $1^{\text {st }}$ line of $v_{n}$

If instead of the first, we fix the $\mathrm{k}$ component we obtain a vector

$$
d^{k}=v_{n} \cdot u^{k}
$$

where $u^{k}$ is the transpose conjugate of the kth line of $v_{n}$. Let $Q^{k}(z)$ be the corresponding polynomial. Then (3) can be expresed as :

$$
P(\omega)=\left[\sum_{k=0}^{L-1} e^{j k \omega} Q^{k+1}(\omega)\right]^{-1}
$$

which shows a relation between MUSIC and the Min-Norm.

Using as alternative to MUSIC, the estimator EV of Johnson and De Graaf [2], we would obtain, instead of (8):

$$
\bar{d}^{k}=v_{n} \cdot \Lambda_{n}^{-1} u^{k}
$$

as vector coefficients of the polynomials $Q(j=1, \ldots, L) . \Lambda_{n}$ is the eigenvalue diagonal matrix. So we define, by analogy with (6), the weighted Min-Norm method vector as:

$$
\dot{d}^{1}=v_{n} \cdot n_{n}^{-1} u^{1}
$$

Later we will make a comparison of the results obtained with (8) and with (11).

\section{3.- A RECURSIVE ALGORITHM}

In previous works [5-7] we proposed an algorithm to update the spectral decomposition of the spatial covariance matrix, when it was modified according to :

$$
\mathbf{R}_{\mathrm{n}}=\alpha \mathbf{R}_{\mathrm{n}-1}+\beta \mathbf{s}_{\mathrm{n}} \mathbf{s}_{\mathrm{n}}^{\mathrm{H}}
$$

this algorithm was used in [7] to implement the Min-Norm method by assuming $\mathbf{R}_{n}$ to be modified twice by snapshot, according to:

$$
R_{n}=\alpha R_{n-1}+\beta s_{n} s_{n}^{H}+\beta J \cdot s_{n}^{*} s_{n}^{\top} J
$$

where $J$ is the exchange matrix and $s_{n}$ is the $n$th snapshot. We begin by decomposing the algorithm into two steps:

$$
\begin{aligned}
& \text { a) } \mathbf{R}_{n}^{\prime}=\alpha R_{n-1}+\beta s_{n} s_{n}^{H} \\
& \text { b) } R_{n}=R_{n}^{\cdot}+\beta J \cdot s_{n}^{*} s_{n}^{\top} J
\end{aligned}
$$

This means that we have to perform two spectral decompositions of matrices with the form given by (1). Let $A_{n}$ represent one of such matrices. Suppose the spectral decomposition of the $A_{n-1}$ matrix be given by:

$$
A_{n-1}=V_{n-1} D_{n-1} v_{n-1}^{H}
$$

Then,

$A_{N}=v_{N-1}\left[\alpha \cdot D_{N-1}+\beta \cdot v_{N-1}^{H} v \cdot v^{H} v_{N-1}\right] v_{N-1}^{H}$

We are going to compute $V_{N}$ and $D_{N}$ such that

$A_{N}=V_{N} \cdot D_{N} \cdot v_{N}^{H} \cdot$ Introducing

$$
\mathbf{w}=\mathbf{v}_{\mathrm{N}-1}^{\mathrm{H}} \mathbf{v}
$$

we reduce the problem to the simplest one: compute the spectral decomposition of :

$$
\alpha \cdot D_{N-1}+\beta \cdot w \cdot w^{H}=U_{N} D_{N} U_{N}^{H}
$$

Once $U_{N}$ is obtained, we can compute $V_{N}$

$$
\mathrm{V}_{\mathrm{N}}=\mathrm{V}_{\mathrm{N}-1} \mathrm{U}_{\mathrm{N}}
$$

We apply the definition of eigenvalue and eigenvector to the matrix in the left side in (17) and with a simple manipulation obtain in the case $\lambda \neq 0$ :

$$
\mathbf{u}=\beta\left(\mathbf{w}^{H} \mathbf{u}\right)[\lambda l-\alpha D]^{-1} v \quad \text { if } \mathbf{w}^{H} \mathbf{u} \neq 0
$$

multiplying both sides in (19) by $w^{H}$ we obtain immediately :

$$
\sum_{i=1}^{M-1} \frac{\left|w_{i}\right|^{2}}{\gamma-d_{i}}+\frac{1}{\gamma} \sum_{i=M}^{L}\left|w_{i}\right|^{2}=\frac{\alpha}{\beta} \quad \text { with } \gamma=\lambda / \alpha
$$

where $M-1$ is the number of non zero entries in $D_{N-1}$. The 
solution of (20) give us $M$ eigenvalues. The corresponding eigenvectors are obtained (aside a normalization) from (19). The eigenvectors corresponding to $\lambda=0$ span the null space. It is not hard to see that these vectors do not interfere necessarily in the formation of $R$ matrix. The computation of $w$ leads, generally speaking, to a vector with $L$ non zero elements. However, as there are infinite bases for the null space we can choose that one with a vector paralell to the component of $v$ (if non null) which belongs to the null space. This means that, if the null space has dimension $\mathrm{k}, \mathrm{w}$ has $\mathrm{k}$ components equal to zero, if $v$ belongs to the previous signal subspace, or $\mathrm{K}-1$, if it has a component in the null space. We proceed as stated in the following:

a) let $t_{0}=v$, and compute a sequence of vectors:

$$
\begin{aligned}
& t_{j}=t_{j-1}-a_{j} \cdot v_{j} \quad j=1,2, \ldots, M-1 \\
& \text { with } a_{j}=\left(t_{j-1}^{H}, v_{j}\right)
\end{aligned}
$$

b) let $\mathbf{r}_{M}=\mathbf{V}+\mathbf{t}_{M-1}$, and compute a new sequence of vectors:

$$
\begin{aligned}
& r_{j}=t_{j+1}-b_{j} \cdot v_{j} \quad j=M-1, \ldots, 2,1 \\
& \text { with } b_{j}=\left(t_{j+1}^{H}, v_{j}\right)
\end{aligned}
$$

c) If $r_{1} \neq 0$, normalize and use it as the Mth eigenvector $\mathbf{v}_{M}$. If $\mathbf{r}_{1}=0$ or $M-1$ is equal to the dimension of the array, there is no change in the dimension of the signal subspace.

d) The components of $w$ will be $w_{j}=\left(a_{j}+b_{j}\right) / 2$, for $j=1,2, \ldots, M-1$ and $w_{M}$ is equal to the norm of $r_{1}$.

Equation (20) is very interesting, since it allows us to understand the behaviour of the eigenvalues, when we add more and more dyads. To implement the spectral decomposition of (12.b) we can apply the described algorithm twice a snapshot, using as entrys $v$ and $\mathrm{Jv}^{*}[7]$. However, this is not very efficient and does not make use of the centrohermitian property of the correlation matrix, which eigenvectors $u$ are such that $u=J u^{*}$. If we substitute as entrys $v$ and $J v^{*}$ by the the eigenvectors of the matrix $v \cdot v^{H}+J \cdot v^{*} v^{\top} J$, we reduce the computational effort in (16) and (18) by a factor around $1 / 2$. Let us compute directly such eigenvectors.

$$
v \cdot v^{H}+J \cdot v^{*} v^{\top} J=u_{1} \cdot u_{1}^{H}+u_{2} \cdot u_{2}^{H}
$$

We obtain, after some manipulation

$$
\mathbf{u}=a / \lambda . v+b / \lambda . J \cdot v^{*} \quad u=u_{1} \text { or } u_{2}
$$$$
\text { As } u=J u^{*}, b^{*}=a=\left(v^{H} u\right)
$$

multiplying (24) on the left by $v^{H}$ and $v^{\top}$ we obtain :

$$
\begin{aligned}
& \lambda . a=a\left(v^{H} v\right)+b\left(v^{H} J \cdot v^{*}\right) \\
& \lambda . b=a\left(v^{\top} J \cdot v\right)+b\left(v^{\top} v^{*}\right)
\end{aligned}
$$

Forcing the determinant of this system to be zero it comes:

$$
\left[\left(v^{H} v\right)-\lambda\right]^{2}-\left|v^{\top} J \cdot v\right|^{2}=0
$$

that we will call characteristic equation of the matrix (23). On the other hand, $(25 . a, b)$ give

$$
\frac{a}{a^{*}}=\frac{v^{H} J \cdot v^{*}}{\lambda-v^{H} v}=\frac{\lambda-v^{H} v}{v^{\top} J \cdot v}
$$

It is not hard to conclude that, aside a normalization, the eigenvector $\mathbf{u}$ can be given by :

$$
\mathbf{u}=\mathrm{e}^{\mathrm{j} \theta} \mathbf{v}+\mathrm{e}^{-\mathrm{j} \theta} \mathrm{J} \cdot \mathbf{v}^{*}
$$

with

$$
2 \theta=\varphi-\arg \left\{\mathbf{v}^{\top} \mathbf{J} \cdot \mathbf{v}\right\}
$$

$\varphi$ being 0 or $\pi$ with the sign of $\lambda-v^{H} \mathbf{v}$.

\section{EXPERIMENTAL VERIFICATION}

\section{Experimental framework}

In order to perform an experimental evaluation of the algorithm, we simulated a system with the following characteristics: Array length: 6 ; number of sources : 2; Center frequency : $1 \mathrm{MHz}$; magnitudes: 1 ; minimum signal to noise ration per source: 10dB;. The eigenvalues were computed by the Newton method. [7].

Simulation results

In the following figures we present the results for two sources: 1 fixed and 1 moving. The DOA were computed every 5 , having processed 2000 snapshots. We set $\alpha=.9$. In fig.1.a and 1.b we see the estimated DOA corresponding to the two sources for the two methods. In figs. 2.a and 2.b we represent the magnitudes of the zeros corresponding to the DOA of the $1^{\text {th }}$ source for both methods. As it can be seen, in the weighted method the zeros are near the unit circle.

\section{CONCLUSIONS}

We have just presented a weigthed version of the MinNorm method. We compared its performance with the usual showing through an example that there is some improvement. We have reported, also, a recursive algorithm to implement the eigendecomposition of recursively generated covariance matrix. The algorithm is simple and its preliminary results are very promising in respect to its numerical properties. On the other hand it can be implemented in a paraliel way. We made use of the centrohermitian property of the autocorrelation matrix to reduce the total numerical cost. 

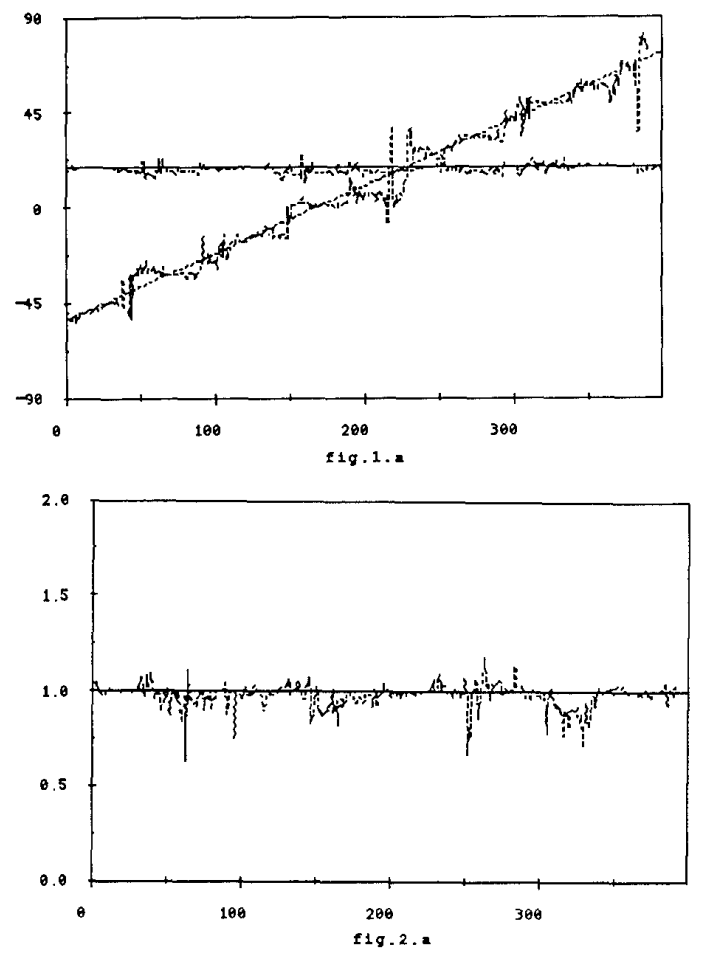

References

[1] Golub, G.H. and Van Loan, C.F. "Matrix Computations Johns Hopkins University Press, 1983

[2] Johnson and De Graaf "Improving the resolution of bearing in passive sonar arrays by eigenvalue analysis," IEEE Trans . on ASSP Vol. 30,No.4, 1982.

[3] Kumaresan,R. and Tufts,D.W.," Estimating the Angles of Arrival of Multiple Plane Waves", IEEE Trans. on Aerospace and Electronic Systems, vol. AES-19, No.1, Jan 1983.

[5] Ortigueira, M. D. "Sobre la Diagonalización Recursiva de Matrices Simétricas", Dep. de Procesado de Señal, ETSE de Telecomunicación, Univ. Polit. de Cataluña, Barcelona, Feb. 1989.

[6] Ortigueira, M. D. and Lagunas, M.A. "Sistema de tracking para arrays de banda estrecha usando SVD" - Proc. of the IV Simposium Nacional de la Union Cientifica Internacional de Radio, Santander, Spain, Sept. 1989.


[7] Ortigueira, M. D. and Lagunas, M.A.," A Recursive Algorithm for the Computation of Eigenvalues and Eigenvectors with application to Array Propcessing", Proc. of the Latvian Signal Processing International Conference, Riga, April, 1990.

[8] Pease, M. C.," Methods of Matrix Algebra," Academic Press, 1965.

[9] Sharman, K.C., " Adaptive Algoritms for Estimating the Complete Covariance Eigenstructure", Proceedings of ICASSP-86, Tokyo.

[10] Schmidt, R.," Multiple Emitter Location and Signal Parameter estimation", Proceedings of RADC Spectral Estimation Workshop, Rome, 1979.

[11] Schreiber,R.," Implementation of Adaptive Array Algorithms", IEEE Transactions on ASSP, vol.34, No.5, Oct. 1986. 